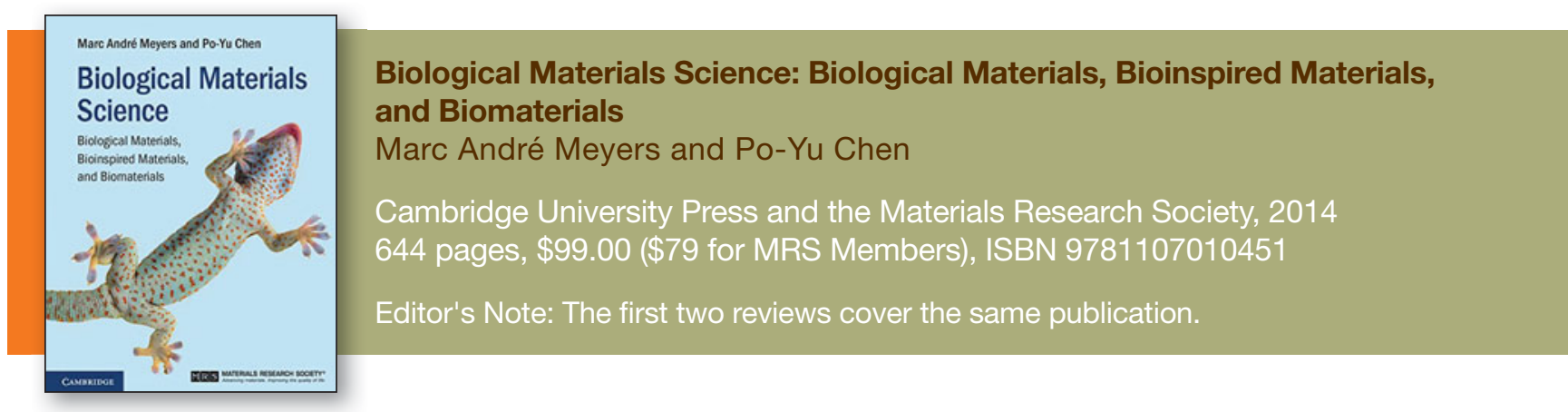

In the interest of transparency, MRS is a co-publisher of this title. However, this review was requested and reviewed by an independent Book Review Board.

$\mathrm{T}_{\mathrm{t}}^{\mathrm{h}}$ his book is an excellent introduction to the field of biomaterials for newcomers to the field or even for the advanced materials scientist who is interested in biomaterials. It is written from the point of view of introducing basic biological concepts. The figures, illustrations, and tables are well conceived, and the reader can easily navigate through the book to get to the sections in which the reader is most interested. The references provide good resources pertinent to the topics covered in the book.

After an introductory chapter 1, Part I, which comprises chapters 2-5, focuses on basic biological principles. This is well written and quite appropriate for engineers and students who are knowledgeable about materials science and engineering, mechanical engineering, or other engineering disciplines, but who have not studied biology to any significant degree. It provides good information to help the reader understand how biological materials are related to biomaterials and the difference between the two. Part I distinguishes this book from many other biomaterials-related textbooks.

Part II consists of chapters 6-11 and is dedicated to presenting and comparing different biological materials. The materials presented include silicate and calcium-carbonate-based composites, calcium-phosphate-based composites, polymer matrix composites, biological polymers, biological elastomers, biological foams, and functional biological materials. This part is very important with respect to the up-and-coming area of biomimetics, which will be a major future area of study in science in years to come. This leads into the last part of the book, which is dedicated to bioinspired materials and biomimetics and contains two chapters.

This book can serve as an excellent upper-level undergraduate course textbook for engineering students interested in bioengineering and biomaterials concentrations. Engineering graduate students involved in biomaterials projects would also be well served by this textbook. The exercises listed at the end of each chapter provide thoughtful questions that challenge the reader's intellect. Overall, the layout of the book is well conceived, and this book will not be outdated for some time, even in the everevolving world of bioinspired science. This book will help materials scientists and engineers of all disciplines dealing with biomaterials understand the complex struggle of biological form and function in their pursuit to advance science and engineering and biotechnology.

Reviewer: Carl J. Boehlert is a professor in the Department of Chemical Engineering and Materials Science at Michigan State University, USA.

$\mathrm{T}$ he union of the physical and biological sciences is, in many respects, one of the most exciting yet challenging aspects of scientific endeavor today. Nowhere is this more evident than in the area of biological materials science and engineering, where many materials scientists and engineers struggle with the complex puzzle of biological form and function, and biologists, in turn, have to deal with the invariably highly quantitative nature of the physical sciences and engineering. Meyers and Chen have delivered a true tour de force that takes the reader, in clear and precise text, from cells to virus-produced batteries. Taking a unique materials science approach, this text introduces students to the basic concepts and applications of materials and biomedical engineering, and prepares them for the challenges of the new interdisciplinary field of biomaterials science.

The book is divided into three partsBasic Biology Principles, Biological Materials, and Bioinspired Materials and Biomimetics. The first part introduces the principles of biology to engineers; the second part presents biological materials along the structural and functional classification created by Ulrike Wegst and Michael Ashby: biopolymers, bioelastomers, foams, and ceramic composites; and the third part discusses more traditional biomimetic designs, such as that of Velcro, in conjunction with new developments that mimic the structure of biological materials at the molecular level, mixing nanoscale with biomolecular designs. Interspersed throughout the book are discussions of biomaterials, such as hip implants, pacemakers, biodegradable materials, and dental implants. Each biomaterial is presented in a focused "box," providing a useful break from the sequence of subjects.

Because the book explains the basic principles of biology, no prior knowledge is required to read this book. Thus, the materials engineer can progress through the book without getting lost in the maze of nomenclature that characterizes biology. The text is supported by approximately 500 figures and plots, since this is a visual topic that needs illustrations to help the reader to grasp 
the concepts. There are also solved problems in the chapters and approximately 120 end-of-chapter exercises. This feature is very helpful for use as a textbook. I am informed by the authors that the Cambridge University Press can provide PowerPoint presentations of the chapters, as well as solved exercises. Consequently, this book should be of great benefit for undergraduate and graduate students in both mechanical engineering and materials engineering who wish to delve into this fascinating subject. Additionally, it is also a valuable reference for researchers in the rapidly expanding fields of the science of biological, bioinspired, and biomaterials. Cambridge University Press is keeping the price at USD\$99 (\$79 for MRS Members), making the book affordable to young scholars.

Reviewer: Robert O. Ritchie is the H.T. \& Jessie Chua Distinguished Professor of Engineering at the University of California-Berkeley, USA.

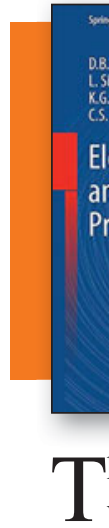

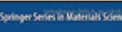

D.8. Srivechmukh

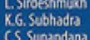

Electrical, Electronic

and Magnetic

Properties of Solids

\section{Properties of Solids}

Electrical, Electronic and Magnetic

D.B. Sirdeshmukh, L. Sirdeshmukh,

K.G. Subhadra, and C.S. Sunandana

Springer, 2014

501 pages, $\$ 179.00$

ISBN 978-3-319-09984-2
Pippard experimental setup are discussed to explain how physical properties are experimentally measured. Basics about semiconductors, including physical properties and their different types, are discussed from a quantum mechanical perspective in the sixth chapter.

Applications of semiconducting devices such as diodes, transistors, and metal oxide semiconductor field-effect transistors, as well as experimental material preparation techniques are very well discussed in the seventh chapter. Different types of magnetism, the associated theory, and the experimental methods to measure magnetic properties are elaborately discussed in the eighth and ninth chapters. An explanation about concepts of domains at the end of the ninth chapter is very interesting, and will give the readers a very clear idea about what happens in magnetic materials in the presence of a magnetic field. In chapter 10, with the help of crystallography and neutron diffraction, different magnetic structures are discussed. Basic components of a neutron diffractometer, an early neutron experimental device (chopper), and a modern-day neutron reactor and their functionalities are pictorially demonstrated. Chapter 11 is dedicated to explaining the fundamentals, theory, and applications of magnetic resonance with the help of experimental tools. Basics and experimental techniques of fast ion conductors with a few example materials are discussed in chapter 12. This chapter will be very interesting for students to learn about the battery and fuel-cell field. The final chapter is dedicated to explain the fundamentals and different types of superconductors along with their applications.

Illustrations in this book that explain the models are very useful to understand concepts. The pictorial representation of random motion of electrons (chapter 2), band filling of electrons at $\mathrm{T}=0 \mathrm{~K}$ (chapter 3), and what happens to the $p-n$ junction when it is biased (chapter 7) are a few examples of clarifying concepts with clear illustrations. Regarding references, the authors might have included more, especially recent ones. In summary, the fundamentals of electrical, electronic, and magnetic materials are well discussed in this book. I strongly recommend this book to all undergraduate and postgraduate students interested in solidstate physics.

Reviewer: K. Kamala Bharathi of the National Institute of Standards and Technology/University of Maryland, USA. such as the cyclotron resonator and the

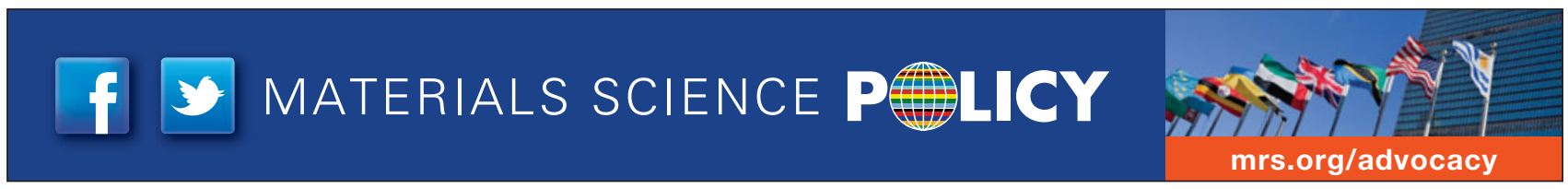

